\title{
Propriocepção na prevenção e tratamento de lesões nos esportes
}

\author{
Proprioception in the prevention and treatment of lesions in sports \\ Cristiane Ferreira Chaskel ${ }^{1}$, Cássio Preis ${ }^{2}$, Luiz Bertassoni Neto ${ }^{3}$

\section{RESUMO}

Introdução: A propriocepção é a capacidade de percepção da posição estática e dinâmica do corpo e seus segmentos. Objetivo: Analisar o foco das publicações atuais e rever o conteúdo sobre avaliação, tratamento e prevenção da propriocepção na recuperação cinética funcional esportiva.

Materiais e Métodos: Revisão de periódicos científicos dos últimos 12 anos, através dos descritores: propriocepção, traumatismos em atletas e fisioterapia; no portal CAPES, além de busca específica em PEDro e COCHRANE. Incluiu estudos que se propunham a avaliar, prevenir ou tratar a propriocepção em lesões possíveis nos esportes, foram excluídos estudos com população menor que 15.

Resultados: Foram encontrados no total de 1412 artigos. Os 43 artigos que atendiam os critérios foram tabulados e organizados por articulação abordada. Equipamentos tecnológicos e instrumentos de baixa complexidade e custo podem ser úteis para avaliar variáveis relativas à propriocepção. Técnicas que objetivam aumento de força muscular associada ao equilíbrio estático e dinâmico parecem contribuir com desempenho do sistema proprioceptivo, melhorando a estabilidade e defesa articular.

Conclusão: A avaliação, tratamento e prevenção da propriocepção demonstrou alta relevância na manutenção do equilíbrio, da estabilidade articular e do controle da força muscular excêntrica sendo de grande valia na recuperação cinética funcional das lesões e desempenho do atleta. As articulações do quadril e punho/mão carecem de estudos a respeito. Prevenção de lesões, comparações entre intervenções, em especial estudos em longo prazo, além de estudos sobre a fisioterapia proprioceptiva na hidroterapia, ou específica a um esporte enriqueceriam o conteúdo científico atual.

Palavras-chave: propriocepção; traumatismos em atletas; fisioterapia.

\section{ABSTRACT}

Introduction: Proprioception is the ability of perception between static and dynamic position of the body and its threads. Objective: To analyze the focus of current publications and review content on assessment, treatment and prevention of proprioception kinetic functional recovery in sports.

Materials and Methods: Review of scientific journals of the past 12 years, through the descriptors: proprioception, athletic injuries and physiotherapy, in CAPES portal, in addition to a specific search in PEDro and Cochrane bases. Included studies were designed to assess, prevent or treat proprioception in possible injuries in sports; we excluded studies with less than 15 people.

Results: We found a total of 1412 articles. The 43 articles that met the criteria were tabulated and organized in conjunction addressed. Technological equipment and instruments with low complexity and cost may be useful to assess variables related to proprioception. Techniques that aim to increase muscle strength associated with static and dynamic balance seem to contribute to the proprioceptive system performance, improving stability and articulate defense.

Conclusions: The assessment, treatment and prevention of proprioception demonstrated high relevance in maintaining balance, joint stability and control of eccentric muscle strength being very important in the recovery kinetic functional injuries and athletic performance. The joints of the hip and wrist / hand lack of studies about it. Injury prevention, comparisons between interventions, particularly long-term studies, and studies on proprioceptive physiotherapy hydrotherapy, or specific to a sport could enrich current scientific content.

Keywords: proprioception; athletic injuries; physical therapy specialty.

\footnotetext{
${ }^{1}$ Fisioterapeuta. Especialista em Fisioterapia Ortopédica, Traumatológica e Desportiva pela Pontifícia Universidade Católica do Paraná (PUCPR), Curitiba, Brasil.

${ }^{2}$ Fisioterapeuta. Mestre em tecnologia em saúde (PUCPR). Coordenador do Curso de Especialização em Fisioterapia Ortopédica, Traumatológica e Desportiva (PUCPR). Docente da Faculdade Dom Bosco e PUCPR.

${ }^{3}$ Fisioterapeuta. Mestre em Pedagogia Universitária pela PUCPR. Especialista em Fisioterapia Desportiva pela SONAFE. Docente da PUCPR e do Curso de Especialização em Fisioterapia aplicada à Ortopedia, Traumatologia e Desportiva (PUCPR).
} 


\section{INTRODUÇÃO}

As informações sensoriais do sistema musculoesquelético incluem a propriocepção e a dor ${ }^{1}$. A propriocepção advêm da integração neural das informações relativas à distensão dos músculos, tensões sobre os tendões, posição das articulações e vibração profunda, permitindo a capacidade de distinguir a posição estática e dinâmica do corpo e seus segmentos $^{1,2}$. Os receptores articulares e tendíneos codificam as informações mecânicas e enviam um potencial de ação através de um axônio periférico até um gânglio dorsal e depois até a medula por um axônio proximal, onde via 0 trato espinocerebeler (propriocepção inconsciente) e trato da coluna dorsal / lemnisco medial (propriocepção consciente), essas informações irão ao sistema nervoso central (SNC) onde serão processadas e irão gerar respostas motoras ${ }^{1,2}$.

Equilíbrio pode ser definido como a manutenção do centro de gravidade do corpo em sua base de apoio ${ }^{3}$. O equilíbrio é um componente importante da habilidade esportiva, pois é envolvido em praticamente todas as formas de movimento ${ }^{2}$ e depende da informação mecânica dos receptores musculares e articulares para manutenção do centro de gravidade ${ }^{2}$.

A estabilidade articular deriva de estruturas estáticas, como ligamentos e cápsulas articulares, que restringem a amplitude de movimento aos limites anatômicos normais, de mecanismos proprioceptivos para o controle, e da força muscular excêntrica, que contribui à estabilidade articular promovendo desaceleração no final do arco de movimento ${ }^{4}$.

$\mathrm{Na}$ prática esportiva o atleta está sujeito há vários tipos de lesões, seja por traumatismo local direto, seja por sobrecarga repetitiva ${ }^{5}$. As lesões resultam em déficits na propriocepção, por causar alterações nas habilidades dos sensores mecânicos articulares e musculares, gerando informações inadequadas para o $\mathrm{SNC}^{2}$

O melhor desempenho esportivo é o do indivíduo sem lesões. Para isso a prevenção se faz necessária. Pouco se utiliza da propriocepção como critério de prevenção, aumentando assim recidivas de lesões em atletas. A maioria dos achados sugere que a propriocepção é importante na recuperação cinético funcional e deve ser realizada tanto como prevenção, como no tratamento de lesões, tendo efeitos consideráveis em curto prazo ${ }^{6}$. Existe evidência de que o treino proprioceptivo reduz a incidência de algumas lesões, sendo que a reeducação proprioceptiva contribuiu muito para a perda de medo no retorno ao trabalho, bem como às atividades desportivas ${ }^{7}$.

O sistema proprioceptivo do corpo humano pode condicionar-se através de exercícios específicos para responder com maior eficácia de forma a melhorar a força, a coordenação motora, o equilíbrio, tempo de reação a determinadas situações e compensar a perda de sensações ocasionadas por uma lesão articular para evitar o risco de que esta volte a se reproduzir ${ }^{2,8,9,10}$. O treinamento proprioceptivo específico é condição obrigatória para a recuperação cinético funcional de lesões, prevenção de reincidências e também pode ser utilizado como treinamento básico para prevenção de lesões ${ }^{4,8,9,10,11,14}$.

O presente estudo visa analisar o foco das publicações dos últimos 12 anos e rever o papel da propriocepção na recuperação cinética funcional esportiva, preventiva e curativa, visto a importância da avaliação, tratamento e prevenção da propriocepção.

\section{MATERIAIS E MÉTODOS}

Este estudo, segundo $\mathrm{Gil}^{15}$ é uma pesquisa exploratória, com base em seu objetivo e uma revisão de periódicos científicos, com base nos procedimentos técnicos utilizados ${ }^{15}$, nos idioma inglês e português, utilizando as palavras-chave: propriocepção (proprioception), fisioterapia (physiotherapy) e traumatismos em atletas (athletic injuries). Realizada no portal CAPES, encontrando 1380 artigos, além de busca específica nas bases Physiotherapy Evidence Database (PEDro), e Cochrane, encontrando 32 artigos, somando o total de 1412 artigos.

Os resumos de todos os artigos encontrados foram selecionados, após leitura exploratória ${ }^{15}$, segundo os seguintes critérios de inclusão: publicação de janeiro de 2000 até maio de 2012, amostras na faixa etária de 12 a 70 anos; que abordassem lesões possíveis em praticantes de esporte, que tivessem como objetivo avaliar, prevenir ou tratar a propriocepção. Foram 


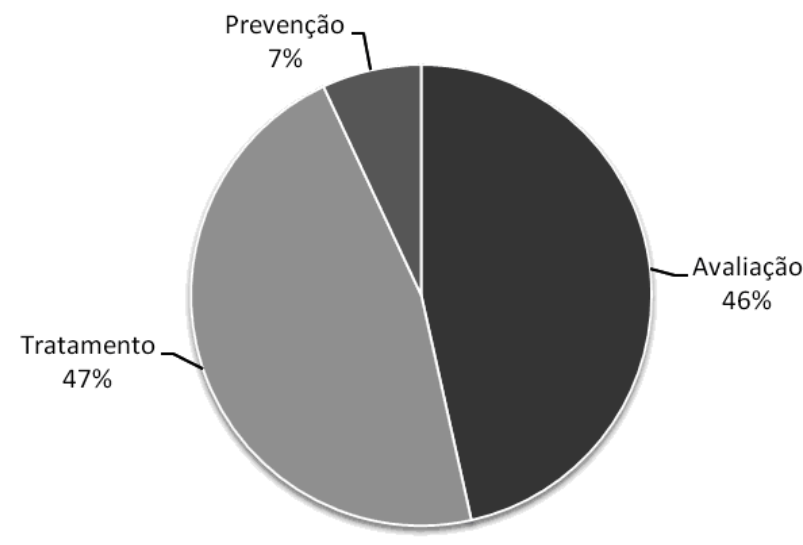

FIGURA 1 - Distribuição por conteúdo abordado.

excluídos estudos com população menor que 15 sujeitos. Chegando a 43 (quarenta e três) artigos que atendiam os critérios adotados (tabela 1).

Todos os artigos foram tabulados no aplicativo Excel do Office Home and Student 2010 da Microsoft nas categorias: título, publicação, tipo de estudo, assunto (avaliação, tratamento ou prevenção), articulações abordadas, objetivos, amostra, métodos, medições, resultados e conclusão. Em seguida foram organizados por conteúdo e topografia da articulação abordada. A partir das tabelas foram geradas figuras ilustrativas e tabelas expositivas.

\section{RESULTADOS}

Foram encontrados 1380 artigos no portal CAPES, além de 32 em busca específica nas bases Physiotherapy Evidence Database (PEDro), e Cochrane, somando o total de 1412 artigos. Os 43 artigos que atendiam os critérios foram tabulados e organizados por articulação abordada.

Dos 43 (quarenta e três) artigos que atendiam aos critérios, 20 (vinte) focaram na avaliação da propriocepção, 20 (vinte) no tratamento e 3 (três) na prevenção de lesões esportivas (figura 1). Do total, 7 (sete) tratavam da propriocepção globalmente, sem focar em articulações específicas, 7 (sete) se referiam a tornozelo, 17 (dezessete) a joelho, 1 (um) a quadril, 5 (cinco) a alguma região da coluna, 4 (quatro) a ombro e 1 (um) a punho/mão (tabela 1).

Foi encontrada uma desproporcionalidade na distribuição do foco da pesquisa, tanto quanto ao

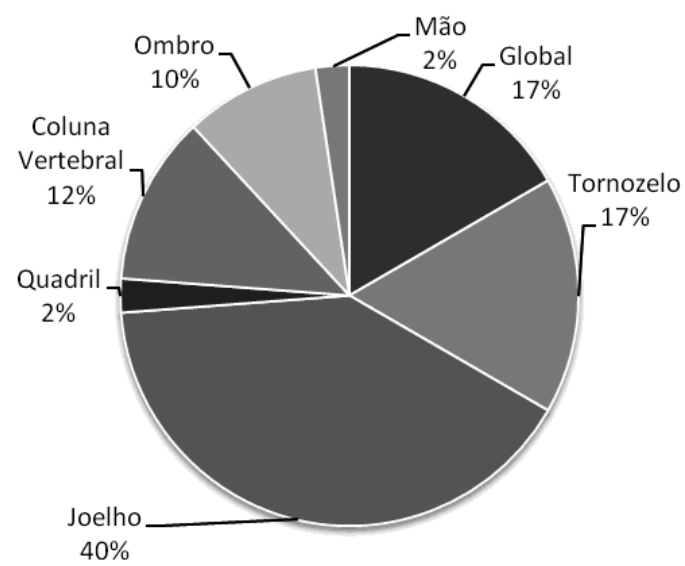

FIGURA 2 - Distribuição por articulação abordada.

conteúdo, quanto às articulações abordadas, demonstrando carência em estudos sobre as articulações do quadril e punho/mão e na prevenção de lesões (figuras 1 e 2).

Tanto equipamentos tecnológicos quantitativos, quanto instrumentos de baixa complexidade e custo podem ser úteis para avaliar variáveis relativas à propriocepção. Para a avaliação do equilíbrio estático é possível utilizar o equipamento NeuroCom Smart Balance Master long-force plate system (NeuroCom International, Inc, Clackamas, Ore), uma plataforma de força com superfície plana e estável, contendo transdutores de força em interface com computador, pode ser testado com olhos abertos e fechados ${ }^{2}$. Outra opção para avaliar a estabilidade postural estática, com boa relação custo-benefício, é o instrumento Balance Error Scoring System (BESS), um método de pontuação do equilíbrio ${ }^{16}$. O equilíbrio estático do joelho pode ser medido pelo KAT 2000, uma plataforma de equilíbrio projetada para treinamento e testes funcionais do sistema de controle neuromuscular ${ }^{10}$.

O equilíbrio semidinâmico foi medido utilizando o Biodex Balance System SD (Biodex Medical Systems, Inc, Shirley, NY), este equipamento é uma plataforma rígida capaz de se mover multidirecionalmente sobre um eixo axial ${ }^{2}$. O equilíbrio dinâmico pode ser aferido a partir de uma modificação do Bass Test of Dynamic Balance, que se baseia em medir a distância de uma série de 10 tentativas de saltar e pousar ${ }^{2}$, do deslocamento vertical e horizontal anteroposterior e laterolateral na plataforma de força ${ }^{18}$ e a partir do Star 
TABELA 1 - Caracterização dos estudos incluídos.

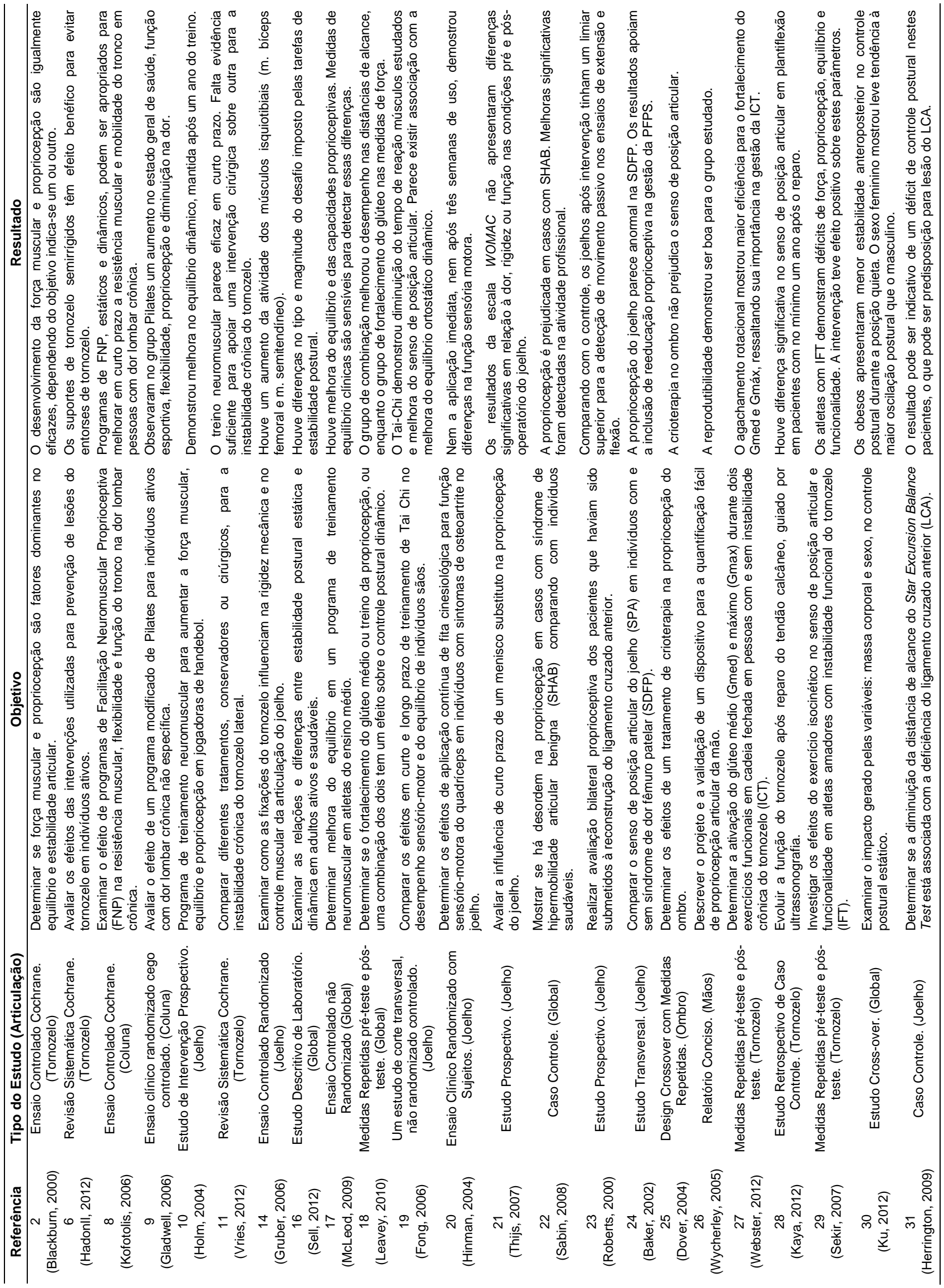


TABELA 1 - Continuação.
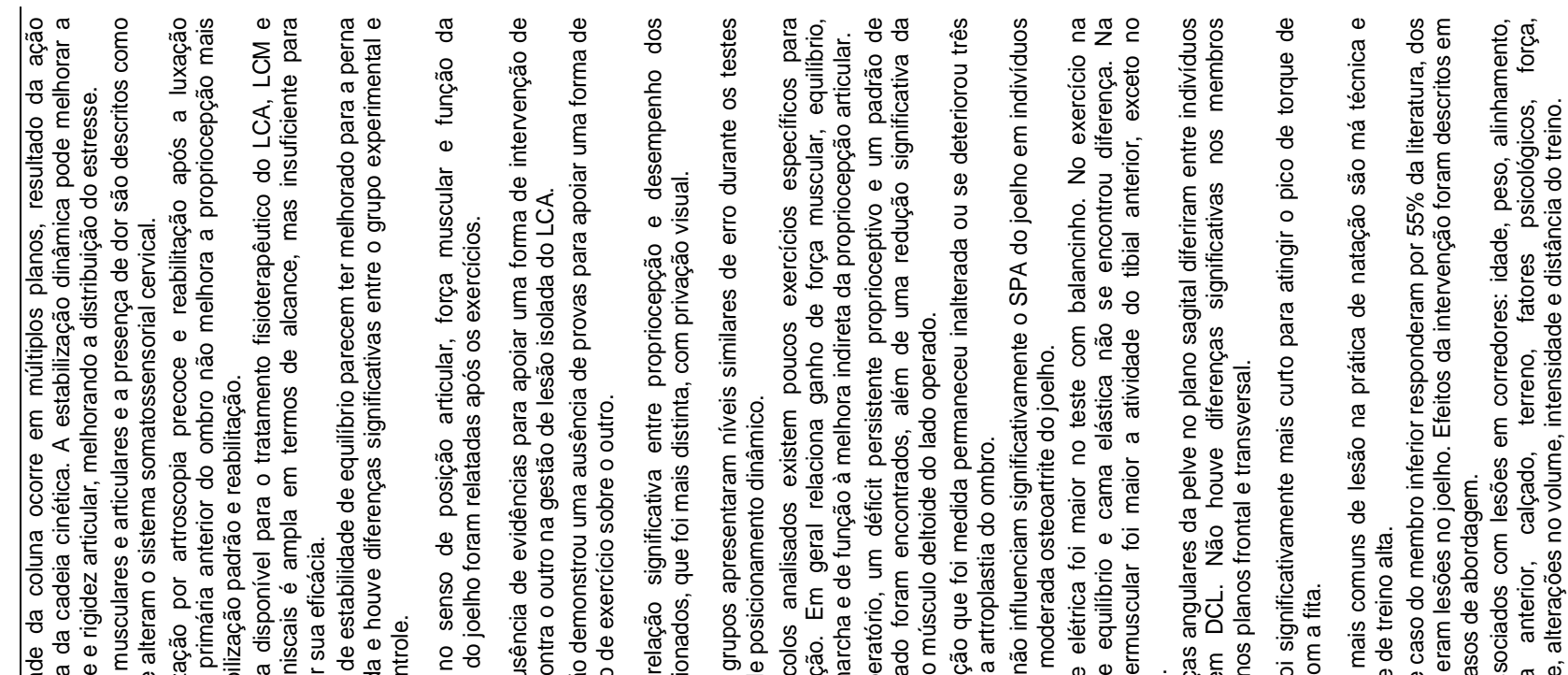

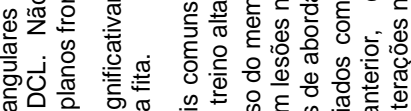
क人

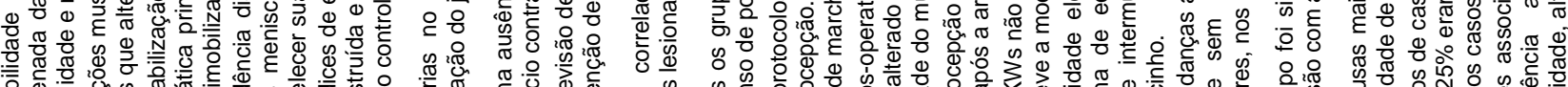

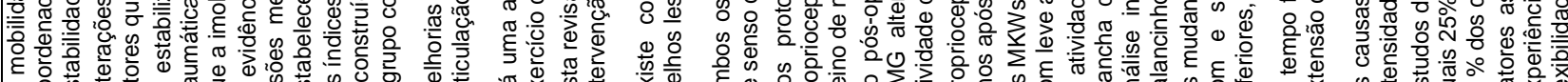

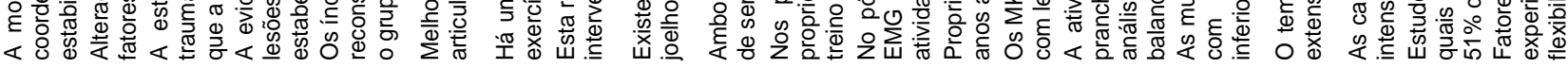

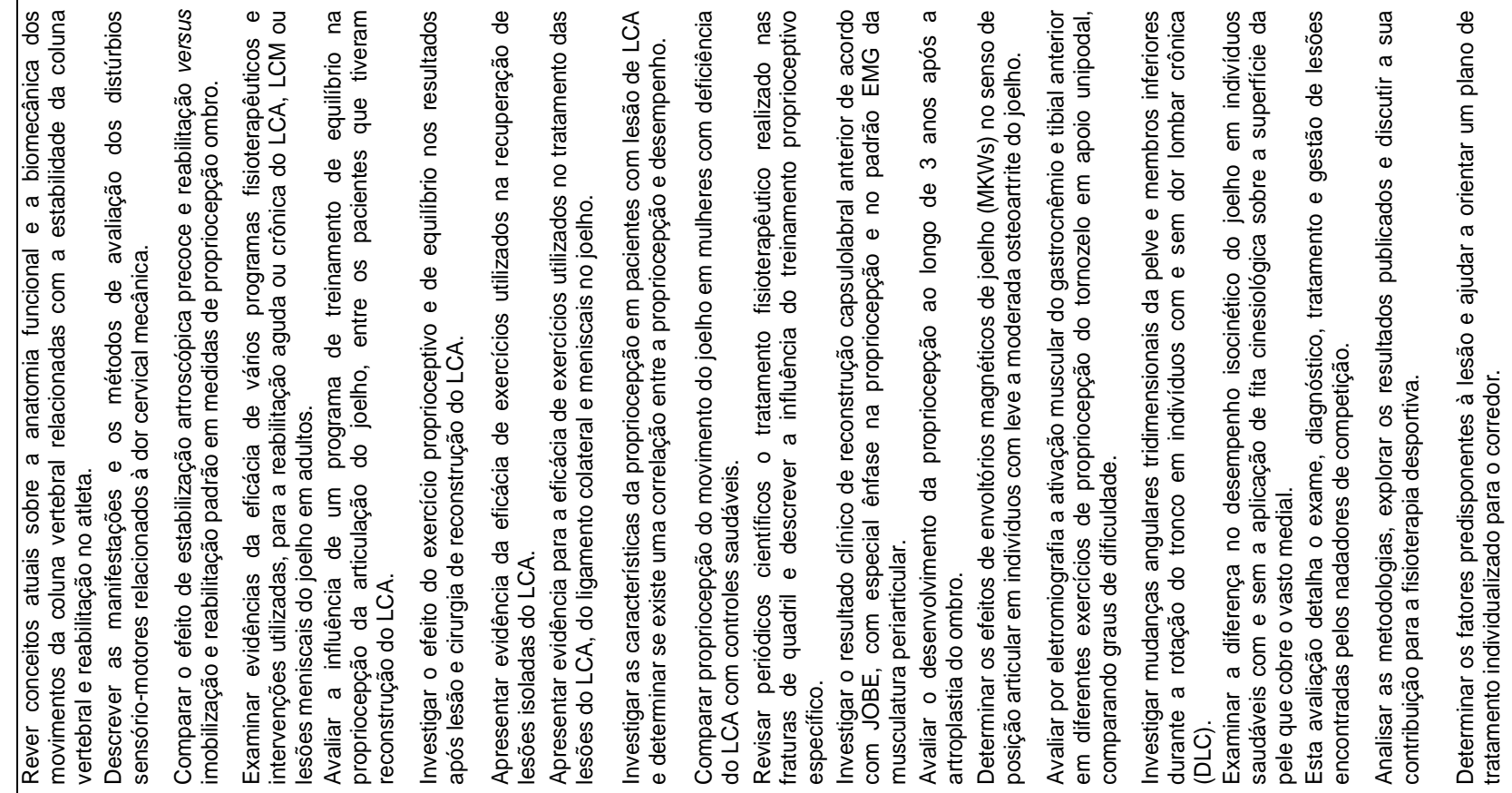

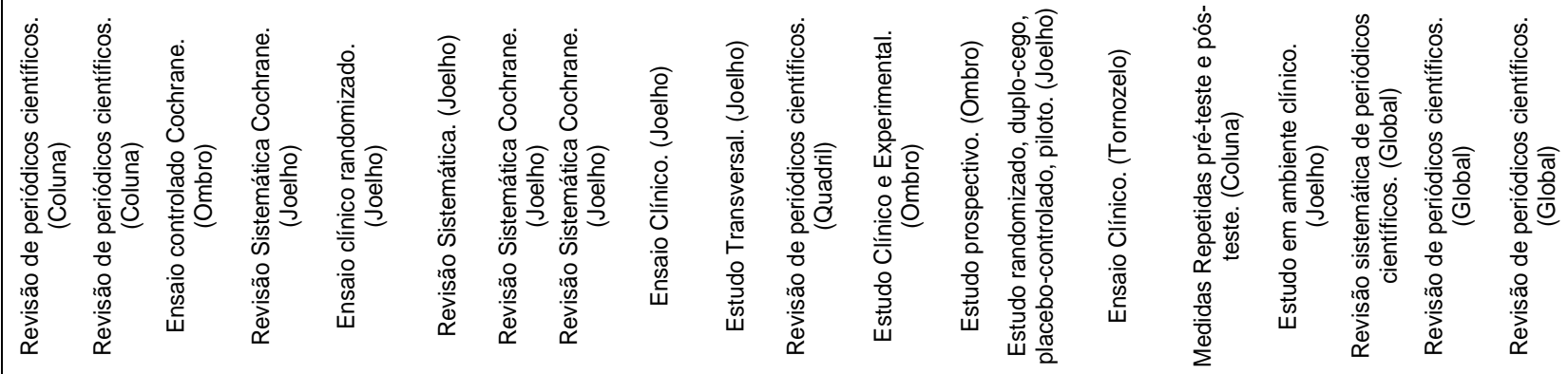

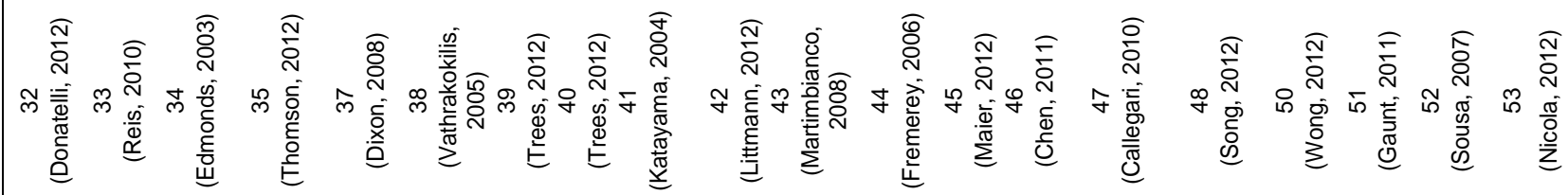


Excursion Balance Test (SEBT), que consiste em oito linhas retas de $120 \mathrm{~cm}$ de comprimento e três $\mathrm{cm}$ de largura, tendo estas retas o início em um ponto único, formando um centro, com angulação de $45^{\circ}$ entre as $\operatorname{retas}^{17,18}$

A latência da contração reflexa (tempo de reação) dos isquiotibiais mediais e gastrocnêmio após perturbação foi medida a partir do erro do ângulo de reposicionamento articular ativo do joelho e o tempo de equilíbrio em uma plataforma de inclinação ${ }^{26}$.

As medidas de propriocepção foram feitas a partir do limiar de detecção de movimento passivo ${ }^{10,20}$. O senso de posição articular pode ser mensurado com o dinamômetro isocinético Biodex System $3^{21,22,23}$. O senso de posição articular do joelho pode ser analisado também com e sem a sustentação de um peso uni e bilaterais usando protocolo validado e confiável, com registros em vídeo de testes da articulação do joelho e testes de resposta sobre posição espacial ${ }^{24}$. O senso de posição articular do ombro dominante foi avaliado utilizando um inclinômetro ${ }^{26}$. Wycherley et al. ${ }^{26}$ descreve um novo equipamento portátil para medir a propriocepção de punhos/mãos, que demonstrou reprodutibilidade.

Para os valores de força muscular da abdução de quadril utilizou-se Sistema de Lafayette: dinamômetro manual para teste muscular (MMTS; modelo 01163 , Instruments Lafayette, Lafayette, $I M^{18}$. Para as medições de força muscular foram utilizados: o Cybex 600010 e o dinamômetro Isocinético KinCom (em 60 $0^{\circ}$ de flexão de joelho ${ }^{20}$. Webster et al. ${ }^{27}$, mediram a média da atividade eletromiográfica do glúteo médio e do glúteo máximo em sua máxima excursão, comparando grupos de indivíduos com e sem instabilidade de tornozelo crônica e exercícios funcionais: agachamento e afundo rotacional. A atividade do vasto lateral foi mensurada através de eletromiografia de superfície durante a descida de degraus ${ }^{20}$.

A função do tornozelo foi mensurada a partir da amplitude de movimento passiva bilateral da dorsiflexão e da plantiflexão, da reprodução ativa de $10^{\circ}$ de dorsiflexão e $15^{\circ}$ de plantiflexão de amplitude de movimento, do pico de torque isocinético concêntrico, em dorsiflexão e plantiflexão de tornozelo durante o salto horizontal e o salto vertical unipodal ${ }^{27}$; do curso do salto unipodal $(S L H C)$; do salto horizontal único e triplo (OLHD-TLHD); dos saltos cruzados em seis metros por tempo (SMHT-CSMHT) e do ângulo do pico isocinético em inversão e eversão, medido excentricamente e concentricamente a $120 \% \mathrm{~s}^{28,29}$

A incapacidade gerada pela dor, a rigidez e a funcionalidade, durante as atividades da vida diária, do joelho afetado, foram medidas pela escala de Western Ontario and McMaster Universities Arthritis (WOMAC) ${ }^{21}$, Arthritis Impact Measurement Scales 2 (AIMS2) e Visual Analogic Scale (VAS) ${ }^{22}$.

A recuperação cinética funcional proprioceptiva pode ser conquistada por meio de diversas técnicas. O equilíbrio e a estabilidade articular dependem da informação mecânica dos receptores musculares e articulares para manutenção do centro de gravidade em cima de uma base de apoio, onde as articulações mais próximas são as do pé e tornozelo, seguidas por joelhos e quadril, exercendo nesta sequência a manutenção do equilíbrio ${ }^{2}$. Relata-se que o índice de massa corpórea e o gênero influenciam no controle postural ${ }^{30}$.

Para a restauração da estabilidade articular é necessário restaurar tanto a propriocepção, quanto a força muscular, pois ambas tem igual influência ${ }^{2,27}$. O fortalecimento funcional, o equilíbrio e os exercícios de estabilização com bolas demonstraram que programas neuromusculares podem aumentar a capacidade proprioceptiva e o equilíbrio ${ }^{17,27}$. Existem concordâncias que os déficits no tempo de reação muscular, na propriocepção e no equilíbrio postural podem ser supridos por treino neuromuscular, tendo eficiência em curto prazo, porém não existem estudos que comprovem seus efeitos em longo prazo ${ }^{10,11}$.

A recuperação cinética funcional do sistema proprioceptivo foi citada como necessária não só em condições crônicas como na síndrome de hipermobilidade articular $^{22}$, na instabilidade de tornozelo ${ }^{27,28}$, na síndrome Patelofemural ${ }^{24,31}$, nos distúrbios posturais e "over use, ${ }^{, 32}$, nas alterações mioarticulares da coluna cervical ${ }^{33}$, na luxação anterior do ombro ${ }^{34}$, como também nas condições agudas que envolvem lesões das estruturas estáticas e dinâmicas envolvidas na propriocepção, foram encontradas nos 
artigos, como nas rupturas ligamentares e meniscais do joelho ${ }^{35,36}$, em especial nas lesões do $\operatorname{LCA}^{23,31,35,37-42}$, na fratura de quadril $^{43}$ e a na mecânica imprópria da coluna $^{32}$. A fisioterapia com ênfase na propriocepção tem papel fundamental após intervenções cirúrgicas, como após artroscopia percutânea para reparo do tendão de Aquiles ${ }^{10,27}$, após a reconstrução do $\operatorname{LCA}^{37}$ e após reconstrução capsulolabral de acordo com a técnica de $\mathrm{JOBE}^{6,34,44,45}$.

Alguns recursos e técnicas foram utilizados para reprogramar direta ou indiretamente a habilidade proprioceptiva. O Tai Chi praticado por mais de um ano demonstrou benefícios no tempo de reação ao desequilíbrio, mensurada pelo desempenho dos isquiotibiais e gastrocnêmios, além de melhorar o senso de posição articular e o equilíbrio ortostático dinâmico. Melhoras no senso de posição podem ser notadas após três meses de prática ${ }^{19}$.

Programas de exercícios isocinéticos demostraram efeito positivo na aquisição funcional comparado ao lado saudável $^{28}$. Assim como o treino de apoio unipodal na aquisição do ganho proprioceptivo, o qual deve ser evoluído em escala progressiva de dificuldade, o equipamento adotado deve ser escolhido com cuidado. Como por exemplo, no balancinho é maior o recrutamento dos músculos tibial anterior e gastrocnêmio, assim como também é maior o grau de dificuldade para manutenção do equilíbrio comparado a cama elástica $^{46,47}$.

O fortalecimento dos glúteos em cadeia cinética fechada, do reto femoral e do semitendíneo ${ }^{14}$, demostrou contribuir no aumento do controle postural de pessoas saudáveis ativas, quando combinado com exercícios proprioceptivos ${ }^{18,27}$.

A bandagem foi popularizada e utilizada em grandeescala nas lesões esportivas. A aplicação da fita cinesiológica não demostrou alterações na geração do pico de torque e trabalho total, porém reduziu o tempo de geração do pico de torque ${ }^{24}$. Falta qualidade metodológica nos estudos encontrados. A influência da bandagem ainda não pode ser comprovada devido a pouca quantidade e baixa qualidade dos artigos ${ }^{10,11,24}$.

O controle neural dos segmentos vertebrais é complexo, pois a mobilidade da coluna ocorre em diferentes planos, resultado da ação coordenada de músculos e articulações. A coluna atua em cadeia cinética, aonde o movimento que ocorre em uma articulação influencia as demais articulações envolvidas na cadeia. A melhora da função é o objetivo principal em pacientes com distúrbios proprioceptivos da coluna, provida através da resistência muscular de tronco e flexibilidade ${ }^{48,49}$, estabilização dinâmica por cocontração dos músculos ${ }^{32}$. Programas estáticos e dinâmicos de facilitação neuromuscular proprioceptiva (FNP) para tronco ${ }^{48}$, e o método Pilates, utilizando exercícios específicos para estabilização de tronco, incorporando movimentos funcionais, podem aliviar a dor crônica não específica em uma população ativa comparando a não intervenção ${ }^{9}$. A presença de alterações dos músculos e das articulações cervicais é descrita como fator que altera o sistema somatossensorial cervical e devem ser considerados também como perpetuantes. As alterações dos sistemas visual, do equilíbrio e proprioceptivo não podem ser desprezadas e devem ser consideradas durante a avaliação fisioterapêutica dos distúrbios cervicais, visto que existe uma integração entre os sistemas ${ }^{33}$.

O tratamento conservador (imobilização) seguido de recuperação cinético funcional foi comparado com a estabilização via artroscopia seguida de recuperação cinético funcional em termos de propriocepção do ombro. Não houve diferença significante entre os grupos. Mostrando que ambos os tratamentos produzem o mesmo efeito em relação à propriocepção ${ }^{6,34}$.

A prevenção tem papel fundamental no desempenho esportivo, levando em consideração as consequências físicas, psicológicas e financeiras e o prejuízo de uma recidiva de lesão. Portanto o melhor rendimento é o anterior a lesão, para isso é importante melhorar o desempenho do sistema neuromuscular, diminuindo os déficits de força muscular, propriocepção, equilíbrio e funcionalidade28 e 0 tempo de reação aos desequilíbrios ${ }^{10,11,14}$.

O tornozelo e pé, responsáveis pela base de apoio de todo o corpo $^{2,28}$, e as estruturas cervicais e cranianas, responsáveis pelo sistema somatossensorial cervical, desempenham papel especial na regência das cadeias musculares que respondem aos desequilíbrios ${ }^{32}$. O 
fortalecimento dos glúteos demostrou contribuir no aumento do controle postural de pessoas saudáveis ativas, mas no tratamento combinado com exercícios proprioceptivos $^{18}$.

Treinos com exercícios de equilíbrio e/ou com propósitos proprioceptivos e de coordenação motora ${ }^{6,50}$, de diversas técnicas tem provido bons resultados na aquisição de força muscular, propriocepção e equilíbrio $^{2,9,10,48}$. Intervenções como modificações em calçados e suportes externos semirrígidos para o tornozelo, podem ser utilizadas para prevenção de lesão ou recidivas, porém deve se levar em consideração os riscos da atividade e o desempenho do jogador ${ }^{6,10}$.

O conteúdo científico sobre propriocepção tem pouco estudo a respeito das comparações entre intervenções, em especial em longo prazo, e estudos sobre a fisioterapia proprioceptiva na hidroterapia, ou específica a um esporte. Não há evidências para apoiar uma forma de intervenção em relação à outra ${ }^{39,40}$. O estímulo bilateral da propriocepção deve ser levado em consideração ${ }^{23}$.

A avaliação, tratamento e prevenção da propriocepção demonstrou alta relevância na manutenção do equilíbrio, da estabilidade articular e do controle da força muscular excêntrica sendo de grande valia na recuperação cinética funcional das lesões e desempenho do atleta. Nos últimos 12 anos o foco maior das publicações sobre propriocepção foi sobre a articulação do joelho, seguida pelo tornozelo, ombro e coluna. As articulações do quadril e punho/mão carecem de estudos a respeito, visto a importância funcional dessas articulações nos esportes. Pesquisas a respeito da prevenção de lesões, comparações entre intervenções, em especial estudos em longo prazo, além de estudos sobre a fisioterapia proprioceptiva na hidroterapia, ou específica a um esporte enriqueceriam o conteúdo científico atual.

\section{REFERÊNCIAS}

1. Lundy-Ekman L. Neurociências: fundamentos para reabilitação. Rio de Janeiro: Elsevier; 2008. p. 89-166.

2. Blackburn T, Guskiewicz KM, Petschauer MA, Prentice WE. Balance and Joint Stability: The Relative Contribuitions of Proprioception and Muscular Strength. J Sport Rehabil. 2000;9:315-28.
3. Nashner LM, McCollum G. The organization of human postural movements: A formal basis and experimental synthesis. Behav Brain Sci. 1985;8(1):135-50.

4. Conduta FL. A importância da propriocepção. Uma revisão bibliográfica. EFDeportes.com. Rev Digital. 2012; 16(165). [capturado em 2012 Out 06]; Disponível em: http://www. efdeportes.com

5. Frisch A, Croisier JL, Urhausen A, Seil R, Theisen D. Injuries, risk factors and prevention initiatives in youth sport. Br Med Bull. 2009;92:95-121.

6. Handoll Helen HG, Al -Maiyah Mohammed A. Surgical versus non-surgical treatment for acute anterior shoulder dislocation. Cochrane Database of Systematic Reviews. In: The Cochrane Library, 2012; 7. Art. No. CF004325. DOI:10.1002/14651858.cd004325.pub1.

7. Domingues MPL, Coimbra FCDEF. Treino proprioceptivo na prevenção e reabilitação de lesões nos jovens atletas. Rev Desporto Saúde. 2008; 4(4):29-37.

8. Kofotolis N, Kellis E. Effects of two 4-week proprioceptive neuromuscular facilitation programs on muscle endurance, flexibility, and functional performance in women with chronic low back pain. Phys Ther. $2006 \mathrm{Jul} ; 86(7): 1001-12$.

9. Gladwell V, Head S, Haggar M, Beneke R. Does a program of Pilates improve chronic non-specific low back pain? J Sport Rehabil. 2006;15(4):338-50.

10. Holm I, Fosdahl MA, Friis A, Risberg MA, Myklebust G, Steen $H$. Effect of neuromuscular training on proprioception, balance, muscle strength, and lower limb function in female team handball players. Clin J Sport Med. 2004;14(2):88-94.

11. Vries JS, Krips R, Sierevelt IN, Blankevoort L, van Dijk CN. Intervention for treating chronic ankle instability. Cochrane Database of Systematic Reviews. In: The Cochrane Library, 2012; 7. Art. No. CD004124. DOI:10.1002/ 14651858.CD004124.pub4.

12. Hughes $T$, Rochester $P$. The effects of proprioceptive exercise and taping on proprioception in subjects with functional ankle instability: a review of the literature. Phys Ther Sport. 2008; 9(3):136-47.

13. Ruiz FT. Propiocepcion: introducción teórica. [capturado em 2005 ago 31]; Disponível em: www.efisioterapia.net.

14. Gruber M, Bruhn S, Gollhofer A. Specific adaptations of neuromuscular control and knee joint stiffness following sensorimotor training. Int J Sports Med. 2006 Aug;27(8):636-41.

15. Gil AC. Como elaborar projetos de pesquisa. $4^{a}$ ed. São Paulo: Atlas; 2006

16. Sell TC. An examination, correlation, and comparison of static and dynamic measures of postural stability in healthy, physically active adults. Phys Ther Sport. 2012; 13:80-6.

17. McLeod TCV, Armstrong T, Miller M, Sauers JL. Balance Improvements in Female High School Basketball Players after a 6 Week Neuromuscular Training Program. J Sport Rehabil. 2009;18:465-81.

18. Leavey VJ, Sandrey MA, Dahmer G. Comparative Effects of 6-Week Balance, Gluteus Medius Strength, and Combined Programs on Dynamic Postural Control. J Sport Rehabil. 2010;19:268-87.

19. Fong $\mathrm{SM}, \mathrm{Ng} \mathrm{GY}$. The effects on sensorimotor performance and balance with Tai Chi training. Arch Phys Med Rehabil. 2006 Jan;87(1):82-7.

20. Hinman RS, Crossley KM, McConnell J, Bennell KL. Does the application of tape influence quadriceps sensorimoto function in knee osteoarthritis? Rheumatology. 2004; 43:331-6. 
21. Thijs $Y$, Witvrouw E, Evens B, Coorevits $P$, Almqvist $F$, Verdonk R. A prospective study on knee proprioception after meniscal allograft transplantation. Med Sci Sports. 2007;17(3):223-9.

22. Sabin N, Baskent A, Cakmak A, Salli A, Uguriu H, Berker E. Evaluation of knee proprioception and efects of proprioception exercise in patients with benign joint hypermobility syndrome. Rheumatol Int. 2008;8:995-1000.

23. Roberts D, Frieden T, Stomberg A, Lindstrand A, Moritz U. Bilateral proprioceptive defects in patients with a unilateral anterior cruciate ligament reconstruction: A comparison between patients and healthy individuals. J Orthop Res. 2000;18(4):565-71.

24. Baker V, Bennell K, Stillman B, Cowan S, Crossley K. Abnormal knee joint position sense in individuals with patellofemoral pain syndrome. J Orthop Res. 2002; 20(2):208-14.

25. Dover G, Powers ME. Cryotherapy does not impair shoulder position sense. Arch Phys Med Rehabil. 2004; 85(8):1241-6.

26. Wycherley AS, Helliwell PS, Bird HA. A novel device for the measurement of proprioception in the hand. Rheumatology. 2005;44(5):638-41.

27. Webster KA, Gribble PA. A comparison of electromyography of gluteus medius and maximus in subjects with and without chronic ankle instability during two functional exercises. Phys Ther Sport. 2012;1-6. DOI: 10.1016/j.ptsp.2012.02.002.

28. Kaya D, Doral MN, Nyland J, Toprak U, Turhan E, Donmez G, Citaker S, Atay OA, Callanghan MJ. Proprioception level after endoscopically guided percutaneous Achilles tendon. Knee Surg Sports Traumatol Arthrosc. 2012. DOI: 10.1007/s00167-0122007-5.

29. Sekir U, Yildiz Y Hazneci B, Ors F, Aydin T. Effect of isokinetic training on strength, functionality and proprioception in athletes with functional ankle instability. Knee Surg Sports Traumatol Arthrosc. 2007 May;15(5):654-64.

30. Ku PX, AbuOsman NA, Yusof A, WanAbas WAB. Biomechanic elevaluation of the relationship between postural control and body mass index. J Biomech. 2012. DOI: 10.1016/j.jbiomech.2012.03.029.

31. Herrington L, Hatcher J, Hatcher A, McNicholas M. A comparison of Star Excursion Balance Test reach distances between ACL deficient patients and asymptomatic controls. Knee. 2009;16(2):149-52.

32. Donatelli R, Dimond D, Holland M. Sport-Specific Biomechanics of Spinal Injuries in the Athlete (Throwing Athletes, Rotational Sports, and Contact-collision Sports). Clin Sports Med. 2012. DOI: 10.1016/j.csm.2012.03.003.

33. Reis FJJ, Mafra B, Mazza D, Marcato G, Ribeiro M, Absalão T. Avaliação dos distúrbios do controle sensóriomotor em pessoas com dor cervical mecânica: uma revisão. Fisioter Mov. 2010;23(4):617-26.

34. Edmonds G, Kirkley A, Birmingham TB, Fowler PJ. The effects of early arthroscopic stabilization compared to nonsurgical treatment on proprioception after primary traumatic anterior discation of the shoulder. Knee Surg Sports Traumatol Arthrosc. 2003;11(2):116-21.

35. Thomson L, Handoll HHG, Cunningham AA, Shaw PC. Phisioterapist led programmes and interventions for rehabilitation of anterior cruciate ligament and meniscal injuries of the knee in adults. Chochrane Database of Systematic Reviews. In: The Cochrane Library, 2012; 07, Art. No. CD001354. DOI: 10.1002 14651858.CD001354.pub2.

36. Dixon J, Trees AH, Howe TE. Exercise for treating isolated meniscal injuries of the knee in adults (protocol for a
Cochrane Review). In: The Cochrane Library, Issue 7, 2012.

37. Vathrakokilis $K$, Malliou $P$, Gioftsidou A, Beneka A Godolias G. Effects of a balance training protocol on knee joint proprioception after anterior cruciate ligament reconstruction. J Back Musculoskelet Rehabil. 2008; 21(4):233-7.

38. Cooper RL, Taylor NF, Feller JA. A Systematic Review of the Effect Of Proprioceptive and Balance Exercises on People With an Injured Or Reconstructed Anterior Cruciate Ligament. Res Sports Med. 2005 Apr-Jun;13(2):163-78.

39. Trees $\mathrm{AH}$, Howe TE, Dixon J, White L. Exercise for treating isolated anterior cruciate ligament injuries in adults. Cochrane Database of Systematic Reviews. In: The Cochrane Library, 2012; 7. Art. No. CD005316. DOI:10.1002/14651858.CD005316.pub4.

40. Trees AH, Howe T E, Grant M, Gray HG. Exercise for treating anterior cruciate ligament injuries in combination with collateral ligament and meniscal damage of the knee in adults. Cochrane Database of Systematic Reviews. In: The Cochrane Library, 2012; 7. Art. No. CD005361. DOI:10.1002/14651858.CD005961.pub3.

41. Katayama M, Higuchi H, Kimura M, Kobayashi A, Hatayama K, Terauchi M, Takagishi K. Proprioception and performance after anterior cruciate ligament rupture. International Orthopaedics (SICOT). 2004; 28:278-81.

42. Littmann $A E$, Iguchi M, Madhavan S, Kolarik JL, Shields RK. Dynamic-Position-Sense Impairment's Independence of Perceived Knee Function in Women With ACL Reconstruction. J Sport Rehabil. 2012;21:44-53.

43. Martimbianco ALC, Polachini LO, Chamilian TR, Masiero D. Efeitos da propriocepção no processo de reabilitação das fraturas de quadril. Acta Ortp Bras. [online]. 2008; [capturado em 2012 Out 06]; 16(2):112-6. Disponível em: http://www.scielo.br/aob.

44. Fremerey $R$, Bosch $U$, Freitag $N$, Lobenhoffer $P$, Wippermann B. Proprioception and EMG pattern after capsulolabral reconstruction in shoulder instability: a clinical and experimental study. Knee Surg Sports Traumatol Arthrosc. 2006;14:1315-20.

45. Maier MW, Niklasch M, Dreher T, Wolf SI, Zeifang F, Loew M, Kasten P. Proprioception 3 years after shoulder arthroplasty in 3D motion analysis: a prospective study. Arch Orthop Trauma Surg. 2012. DOI 10.1007/s00402012-1495-6.

46. Chen CY, Fu TC, Hu CF, Hsu CC, Chen CL, Chen CK. Influence of magnetic knee wraps on joint proprioception in individuals with osteoarthritis: a randomized controlled pilot trial. Clin Rehabil. 2011;25(3):228-37.

47. Callegari B, Resende MM, Ramos LAV, Botelho LP, Albuquerque AS. Atividade eletromiográfica durante exercícios de propriocepção de tornozelo em apoio unipodal. Fisioter Pesq. 2010;17(4):312-6.

48. Song AY, Jo HJ, Sung PS, Kim YH. Three-dimensional kinematic analysis of pelvic and lower extremity differences during trunk rotation in subjects with and without chronic low back pain. Physiotherapy. 2012; 98(2):160-6.

49. Lephart SM, Pincivero DM, Giraido JL, Fu FH. The Role of Proprioception in the Management and Rehabilitation of Athletic Injuries. Am J Sports Med. 1997;25(1)130-7.

50. Wong OMH, Cheung RTH, Li RCT. Isokinetic knee function in healthy subjects with and without Kinesio taping. Phys Ther Sport. 2012; DOI:10.1016/j.ptsp.2012.01.004.

51. Gaunt T, Maffulli N. Soothing suffering swimmers: a systematic review of the epidemiology, diagnosis, treatment and rehabilitation of musculoskeletal injuries in competitive swimmers. Br Med Bull. 2011;7:1-44. 
52. Sousa JP, Cabria J, Donaghy M. Case research in sports physiotherapy: a review of studies. Phys Ther Sport. 2007; 8:197-206

53. Nicola TL, Shami A. Rehabilitation of Running Injuries. Clin Sports Med. 2012;31:351-72.

\section{Endereço para correspondência:}

Cristiane Ferreira Chaskel

Rua Ambrósio João da Silveira 288

Florianópolis/SC - CEP 88066-250

Telefone: +55 4899099920

E-mail: crischaskel@hotmail.com 